\title{
Methods for Leveraging a Health Information Exchange for Public Health: Lessons Learned from the NW-PHIE Experience
}

\author{
${ }^{1}$ Trebatoski M, ${ }^{2}$ Davies J, ${ }^{3}$ Revere D, ${ }^{1}$ Dobbs D \\ ${ }^{1}$ Science Applications International Corporation, McLean, VA \\ ${ }^{2}$ Inland Northwest Health Services, Spokane, WA \\ ${ }^{3}$ Center for Public Health Informatics, University of Washington, Seattle, WA
}

\section{Abstract:}

The intent of this article is to provide public health and health information exchanges (HIEs) insight into activities and processes for connecting public health with clinical care through HIEs. In 2007 the CDC issued a Request for Proposal (RFP) for the "Situational Awareness through Health Information Exchange" project. The project's goals are to connect public health with health information exchanges (HIEs) to improve public health's real-time understanding of communities' population health and healthcare facility status. This article describes the approach and methodology used by the Northwest Public Health Information Exchange to achieve the project's goals. The experience of the NWPHIE Collaboration provides an organizational and operational roadmap for implementing a successful regional HIE that ensures secure exchange and use of electronic health information between local and state public health and health care entities.

Keywords: Data Collection; Electronic Health Records; Health Information Exchange; Information Management; Information Services; Medical Record Linkage; Public Health; Public Health Informatics

\section{Introduction}

In 2007 the CDC issued a Request for Proposal for the "Situational Awareness through Health Information Exchange" project which aims to connect public health with health information exchanges (HIEs) to improve public health's real-time understanding of communities' population health and healthcare facility status. A team consisting of five participants was assembled by the Science Applications International Corporation (SAIC) to form the Northwest Public Health Information Exchange (NW-PHIE): Inland Northwest Health Services (INHS); Washington State Department of Health (WA DOH); University of Washington Center for Public Health Informatics (UW CPHI); Spokane Regional Health District (SRHD); and Idaho Department of Health and Welfare (ID DOHW). More background on the process conducted by SAIC to recruit project participants can be found in the article, "Northwest Public Health Information Exchange's Accomplishments in Connecting a Health Information Exchange with Public Health" in this issue. 
SAIC received a contract award from CDC in early 2008 and began the process of creating collaborative relationships among NW-PHIE participants. The goal was to make sure that all NW-PHIE participants understood their roles and responsibilities and that effective lines of communication were developed.

\section{Methods}

To provide a forum for effective project communication bi-weekly meeting were established where the project leads from each of the member organizations discuss project priorities, activities, risks and issues. This group sets the vision and direction for NW-PHIE. Implementation teams are assigned to carry out specific project activities and hold their own working meetings. A comprehensive project plan coordinates all project activities and tracks project progress. A project collaboration portal was established to share information such as work products, deliverables, project plans and status reports.

Collecting Clinical Data and Making it Useful to Public Health: To realize the potential of tapping into INHS' rich set of clinical data to improve public health surveillance and situational awareness the NW-PHIE project team developed a structured methodology for defining public health's functional and data requirements and implementing the needed technology solution. This methodology is depicted in Figure 1 below.

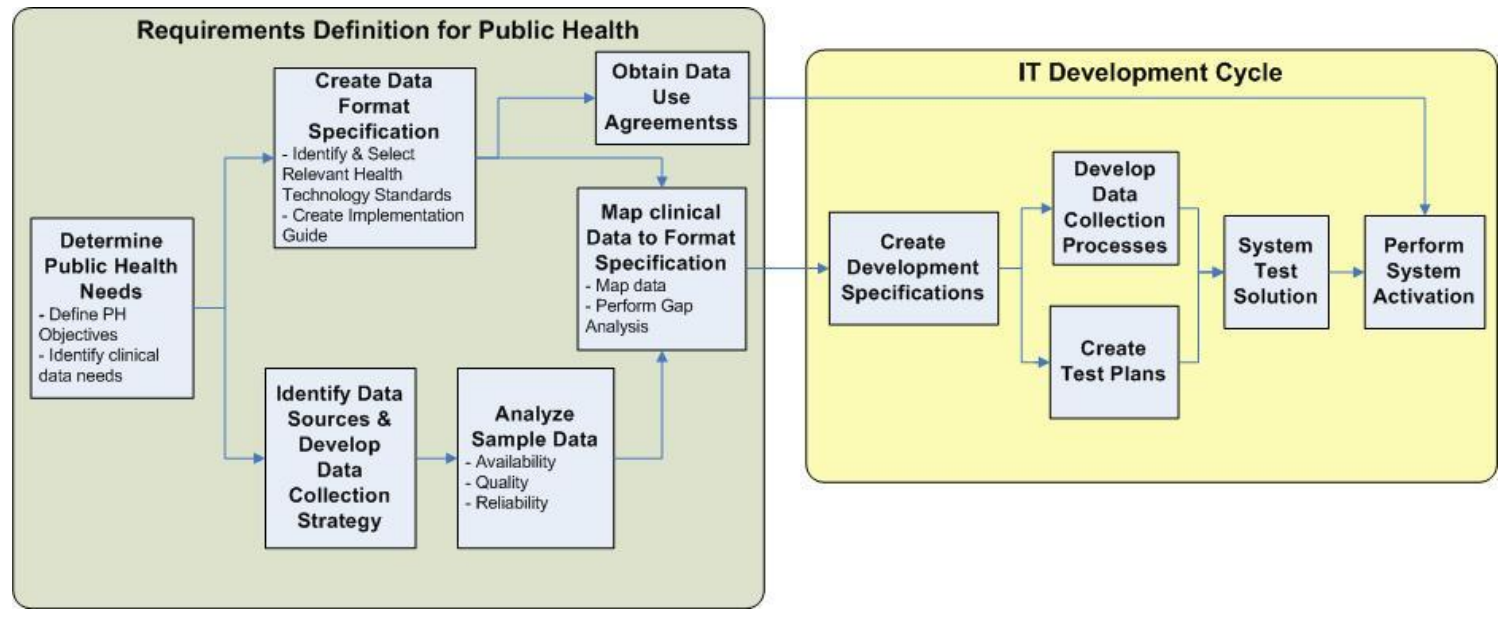

Figure 1. NW-PHIE's Requirement's Definition and IT Development Methodology

Through the use of a structure requirements definition and IT implementation methodology NW-PHIE created a repeatable and efficient process that reduced project costs and risks. Our methodology helps ensure that the proper clinical data is collected and sent to public health and that public health can extract value from the data.

Requirements Definition for Public Health: We began with documenting public health's requirements for collecting clinical data from HIEs. To define project requirements, epidemiologists, public health stakeholders and informaticists documented answers to a fairly straightforward set of questions, including: 
1. What are the over-arching objectives public health is trying to achieve by collecting information from HIEs?

2. What questions will public health try to answer with the collected data?

3. What clinical information is needed to answer public health's questions?

4. What are the timeliness, quality and reliability requirements of the data?

5. How does the data need to be analyzed to answer public health's questions?

NW-PHIE's initial efforts were to capture syndromic surveillance data from INHS member hospitals and provide this information to public health. The starting points for NW-PHIE's syndromic surveillance requirements were the American Health Informatics Community's (AHIC) Biosurveillance Use Case and the MBDS.

The Biosurveillance Use Case provides requirements for the transmission of pseudoanonymized ambulatory care, inpatient and emergency department (ED) visit, utilization, and lab result data from health care organizations to authorized public health agencies with less than one day lag time. Pseudo-anonymization removes patient identifying characteristics from the data and tags the patient level data with a system generated number (pseudo-anonymized identifier). In a public health emergency, authorized pubic health officials can request that the healthcare organization re-identify the patient using the pseudo-anonymized identifier. The Biosurveillance Use case identifies a Minimum Biosurveillance Data Set (MBDS) that contains the clinical and resource utilization data elements that are deemed the minimum list needed to support local, state and federal public health syndromic surveillance functions. These documents provided a solid foundation of requirements and a starting clinical data set for the Situational Awareness project.

NW-PHIE augmented the requirements derived from the AHIC documents with additional requirements from local and state public health agencies. Within Washington State outbreak investigations are initiated by the Local Health Jurisdiction (LHJ). The WA DOH assist LHJ staff by facilitating testing done through the state public health laboratory and/or CDC as well as provide coordination and/or staffing support with outbreaks that involve multiple LHJs or other states. Critical to timely management of outbreaks is early identification of the outbreak along with case identification. Many outbreaks of public health interest are not notifiable based upon case-based mandatory reporting by health care providers, health care facilities or laboratories. Common pathogens that are not reportable as isolated cases would include influenza, varicella, RSV, and norovirus. WA State and LHJ's were very supportive of using automated surveillance systems utilizing HIE data to improve public health response time in identifying outbreaks. Being able to then identify cases based upon being associated with a syndromic surveillance/notifiable condition cluster would provide LHJs and the WA DOH more time to identify the source of the outbreak as well as additional time for contact investigation/management.

The H1N1 epidemic provided a test case for confirming our syndromic surveillance requirements. During this epidemic public health wanted to understand not only the size and spread of the epidemic but also the severity of illness, the rate and efficacy of influenza vaccinations, and instance of influenza in sensitive groups such as pregnant women. NW-PHIE assembled epidemiologists, informaticists, and ED nurses to discuss 
and design how these additional public health data requirements were collected in the clinical setting and how they could be sent to public health.

During the life of the project a public health requirements and data collection document has been maintained. This document describes all clinical data collected across patient types (ED, inpatient and ambulatory care) and serves as a data dictionary for the clinical data being sent to public health.

Identifying Data Sources and Developing a Data Collection Strategy: After documenting the clinical data of importance to public health NW-PHIE created a strategy and process for collecting each type of data by identifying and analyzing existing information stores (i.e., hospital and laboratory information systems) and information flows within INHS' HIE. Common sources of data include Health Level 7 (HL7) admission/discharge/transfer (ADT), Orders and Results messages from participating data sources. In addition, HL7 messages from abstracting and financial systems provided data that is not routinely sent in with ADT based messages.

For a preponderance of the syndromic surveillance data the strategy was to subscribe to existing HL7 clinical data information flows. Custom extracts were required for some of the data types. Table 1 describes the types of syndromic surveillance data NW-PHIE collects and our collection method.

Table 1. Syndromic Surveillance Data Types and Methods of Collection

\begin{tabular}{|c|c|}
\hline Data Type & Method of Collection \\
\hline Base Facility & $\begin{array}{l}\text { Generally static and submitted at baseline. Updated } \\
\text { as necessary. }\end{array}$ \\
\hline $\begin{array}{l}\text { Daily Facility Summary (reflects the } \\
\text { current status of the facility to help } \\
\text { identify developing conditions and } \\
\text { resource capacity) }\end{array}$ & $\begin{array}{l}\text { Creation of nightly census reports as well as custom } \\
\text { extracts from a community-wide resource utilization } \\
\text { system. }\end{array}$ \\
\hline $\begin{array}{l}\text { Deidentified Patient Demographics (for } \\
\text { example, gender, age, zip, state) }\end{array}$ & $\begin{array}{l}\text { Captured through HL7 Admit/Discharge/Transfer } \\
\text { (ADT) transactions. }\end{array}$ \\
\hline $\begin{array}{l}\text { Clinical (for example, patient class, chief } \\
\text { complaint, clinical diagnosis, billing } \\
\text { diagnosis, temperature, pulse oximetry, } \\
\text { discharge disposition) }\end{array}$ & $\begin{array}{l}\text { Obtained by monitoring HL7 messages and facility } \\
\text { identifier. Use of the pseudo-anonymized linker has } \\
\text { been associated with the clinical data element record. } \\
\text { Additional data elements have been obtained through } \\
\text { the use of system extracts where HL7 messages are } \\
\text { not supported by the source systems (e.g., ED clinical } \\
\text { diagnosis). }\end{array}$ \\
\hline $\begin{array}{l}\text { Laboratory Orders (for example, ordered } \\
\text { procedure name and code) }\end{array}$ & Obtained by monitoring HL7 order messages. \\
\hline $\begin{array}{l}\text { Laboratory Results (for example, ordered } \\
\text { data/time, laboratory, test name, test } \\
\text { results, result status) }\end{array}$ & Obtained by monitoring HL7 result messages. \\
\hline
\end{tabular}


An overview of the information exchanged between the INHS and public health is provided in Figure 2. This view reflects the work which is currently underway to convert the transmission of data to CDC using the secure data transport capabilities within the NHIN CONNECT gateway.

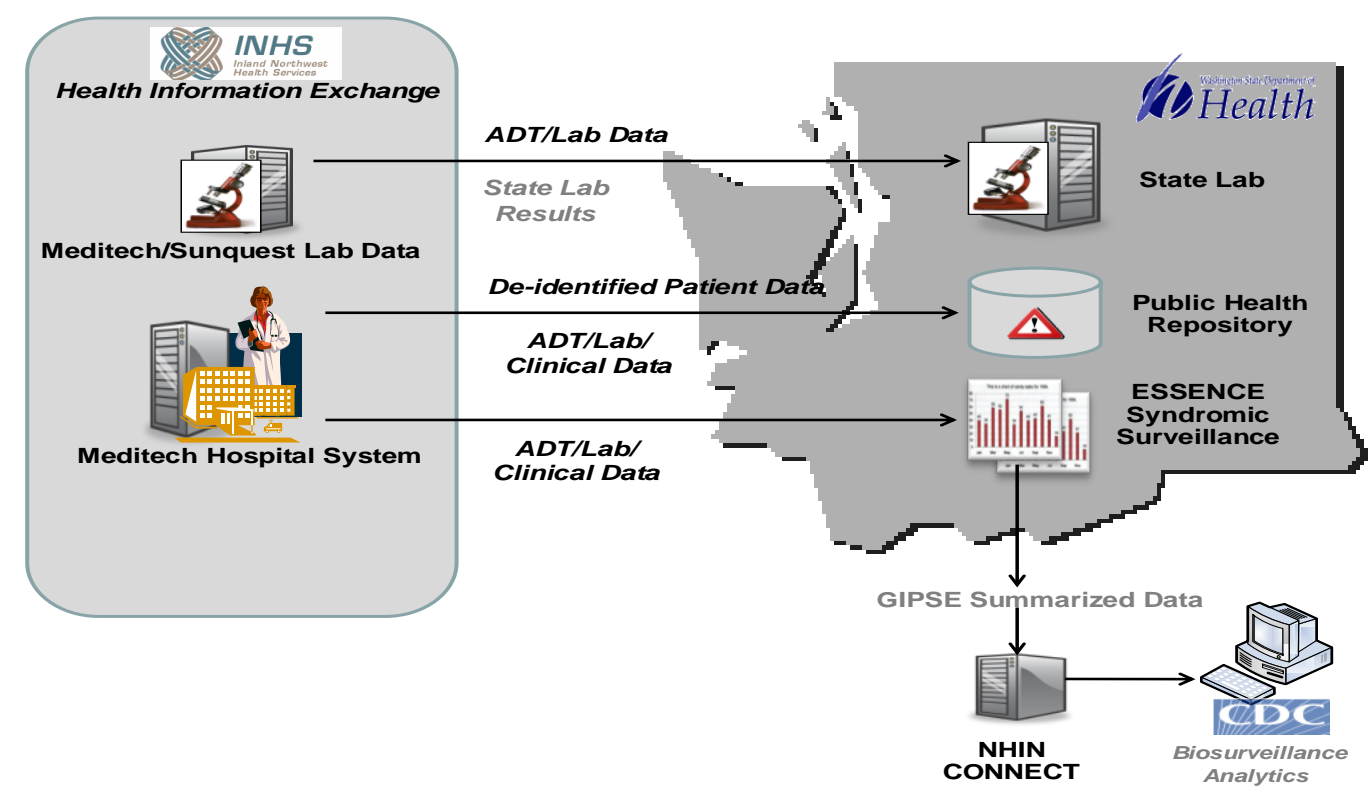

Figure 2. Overview of information flow between INHS and public health

Creating the Data Format Specification: An important step in capturing the requisite clinical information from INHS' HIE was developing a data format specification that describes how the clinical data will be transmitted to public health. Best practices for sharing clinical data are based on sending the clinical data using accepted industry standards such as those from HL7 or Clinical Data Interchange Standards Consortium (CDISC) and encoding those data using standard-based terminology.

The format NW-PHIE used for sending syndromic surveillance data to WA DOH was based on the Health Information Technology Standards Panel (HITSP) Interoperability Specification 02 (IS02) for Biosurveillance which covers the data elements in the MBDS described above. In particular, we decided to implement the HL7 message components for HITSP IS02 as these complemented our data collection strategy of filtering HL7 messages for the MBDS data elements.

HITSP IS02 is an overarching framework for biosurveillance that consists of a complex array of documents that reference a multitude of HITSP capabilities, service collaborations, transaction packages, transactions and components. IS02 also references Integrating the Healthcare Environment (IHE) profiles and base HL7 standards. Because of the complexity of the IS02 specification, NW-PHIE took the lead, in collaboration with the Indiana and New York HIE project teams, for developing a concise implementation guide that pull together all the disparate information from the ISO2 specification into one document, the "HITSP Biosurveillance Message Implementation Guide - HL7 Version 2.5". This document includes requirements for specific 
administrative, demographic and clinical care information and the sharing of this data with public health organizations to support syndromic surveillance needs.

Analyzing Sample Data and Mapping to the Data Format Specification: We performed a detailed review of a sampling of production-like HL7 V2.1 and V2.3 messages for each of the data sources being considered. This allowed us to evaluate the availability of data elements and coding practices and provided insights into data quality and reliability.

We noted differences in data availability and quality based on patient class (inpatient, ED, outpatient), clinical documentation processes and operating procedures. These differences varied from facility to facility and necessitated some facility-based variation in data collection practices.

Based on this analysis, patient filtering logic (partially derived from AHIC Biosurveillance Use Case and the HITSP Biosurveillance Messaging Guide) was developed to screen out certain types of patients and encounters including preadmissions, recurring patients (e.g., dialysis patients), obstetric and psychiatric patient visits. This logic was based on analyzing specific fields in the HL7 messages; the patient class (HL7 field PV1-2), patient type (PV1-18) and patient location (PV1-3) fields. These filtering criteria were then reviewed and validated for each of the INHS hospitals implementation.

Next, we mapped the data fields in the HL7 messages to the implementation guide developed as part of the initial requirements definition process. This provided the necessary specifications to allow mapping of the clinical care information provided by the various data sources to the required message standards and terminology to meet the data output specifications.

Obtaining Data Use Agreements from Facilities: The facilities that participate in the INHS network do so for the purposes of delivering improved and better coordinated health care. Each facility signs agreements upon entering the network that support common data use, access and security policies. Those standard agreements do not address electronic submission of data to public health agencies. While INHS had delivered health care data to public health agencies in the past, it had been limited to very circumscribed situations such as birth records and disease registries or ad hoc one-time data requests related to a specific public health study. In each of those cases INHS developed a customized data use agreement with each facility wanting to submit data to public health, focused specifically on the elements of that particular data request.

NW-PHIE presented a very different scenario. Hospitals would be asked to regularly submit a large data set containing information that was not mandated by any state or county law or regulation. INHS did not have the authority to release the data without full support and signed data use agreements from each participating facility. This was the first syndromic surveillance system many of the hospitals had been asked to participate in. While they were not unwilling to support the project, they did need a clear understanding of the project's purpose and scope and comprehensive assurances that the patient data they held in trust would not be compromised. 
INHS worked with epidemiologists from the Spokane Regional Health District (SRHD) to develop a document that explained the project and also clarified how it was authorized (although not mandated) under existing state regulations. INHS staff then met with representatives of the Health Information Management (HIM) office and infection control staff at each hospital to discuss the project and answer questions. These meetings were most effective when an epidemiologist from the SRHD also participated. The SRHD epidemiologists already had a strong relationship with the hospital staff from prior public health investigations and helped add credibility to the request for data.

The data use agreement itself was designed with standard language authorizing INHS to deliver data to public health agencies on behalf of the participating facility for a period of time stipulated by the facility. Specific data elements to be released and any pertinent methodologies were included as an appendix. This approach allowed senior management from the hospital to sign the overall data use agreement and other staff, usually the HIM director, to sign the appendix and any updates to the appendix over time.

Information Technology Development Cycle: Our requirements definition phase made sure we clearly understood public health's needs for collecting clinical data and the strategy we would employ to collect those data. During the IT development cycle we finished our technical design and developed the needed technology processes for collecting and standardizing the relevant data. Our process design for collecting the clinical data is depicted in Figure 3 below.

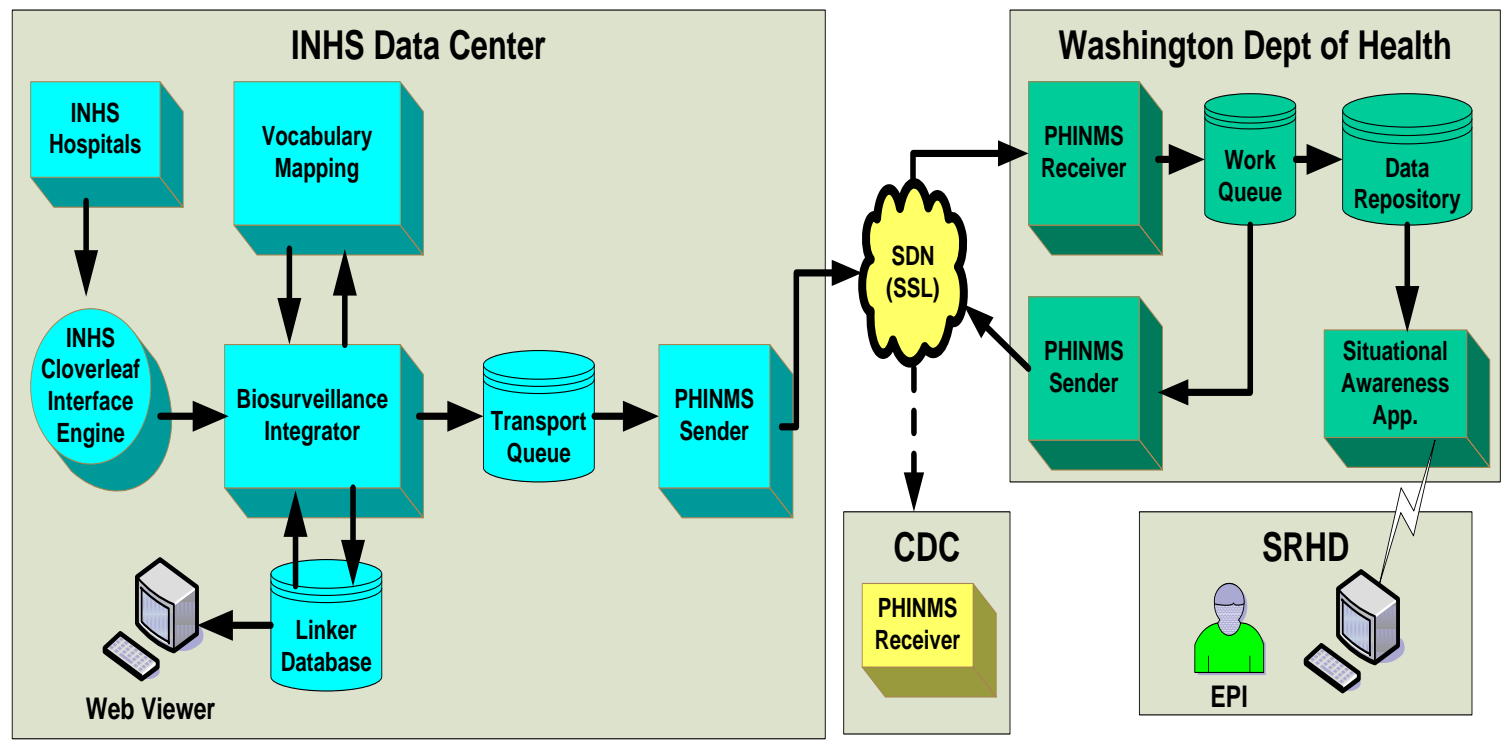

Figure 3. INHS Architectural View of Biosurveillance Solution

Creating the Development Specifications and Process: We created development specifications for each of the components listed in Figure 4. This gave us a very specific understanding of how the different system components interacted with each other 
including: input format and content; processing logic; and out format and content. This holistic design process enabled us to efficiently develop processes and ensured that once all process components were assembled they would reliably work together to collect and transmit the requisite clinical data to public health.

Developing the Data Collection Processes: SAIC developed an integration engine tool known as the Biosurveillance Integrator that receives HL7 messages in real time from INHS's Cloverleaf integration engine via TCP/IP. INHS Cloverleaf engine performs some filtering of messages to limit the number of data elements exchanged and to exclude patient visits which are not routinely associated with an encounter involving an infectious agent (ex. re-occurring visit for physical therapy). INHS also performs filtering of the laboratory data to provide a desired subset of the lab orders and results which are valuable in the identification of infectious diseases, where the approach is to accept all results from the hospital lab that contain these results, even though this approach may result in capturing data beyond the scope of what is desired. INHS provides the data through HL7 messages and several flat file data extracts to populate the Admit/Discharge/Transfer (ADT), Observation Result Message (ORM), Observation Result Unsolicited (ORU), daily census, facility utilization and clinical data to the Biosurveillance Integrator for message transformation.

The Biosurveillance Integrator takes these input data streams and transforms them into well-formed HL7 messages that conform to the HITSP biosurveillance implementation guide. This transformation process includes removing extraneous information such as patient identifiers, personally identifiable information and unneeded clinical information. The process also standardizes the vocabulary using a set of mapping tables. Currently these vocabulary mapping tables are maintained by a programmer. Our plans call for the implementation of a full-fledged vocabulary server so that an end user can maintain the vocabulary mapping process.

The output HL7 messages are encoded with a system generated patient identifier that allows public health department to reassemble the syndromic surveillance messages for a given individual without having to know the facility's actual MPI and visit number. The Biosurveillance Integrator stores a cross-reference between the facility's patient Master Patient Index (MPI) and the system generated patient identifier in a database. HIMs have access to this database and can re-identify patients to public health officials when an authorized request is received.

The output HL7 biosurveillance messages are stored into a file message queue that is picked up every 15 minutes by a Public Health Information Network Messaging System (PHINMS) which compresses, encrypts and digitally signs them before transporting them to the WA DOH over the internet. The WA DOH unpacks the messages and puts them into a work queue.

Creating the Test Plans: To help ensure objective, credible, timely and high quality work, the NW-PHIE team utilized a combination of project management, integration development, and quality control strategies and techniques. A key project management strategy has been to develop detailed test plans based on public health requirements, the 
HITSP biosurveillance implementation guide and our system design specifications. These have given us a proven and repeatable set of processes for system testing our data collection processes and for certifying data feeds from hospitals as they are activated.

To develop our test plans we created a matrix of all types of input data by patient class (ED, inpatient and outpatient) and input format (ADT, ORUs, flat files, etc.). We developed test cases within each of those data types based on our data mapping and development specifications and documented expected results for each test case. Our testing procedures defined pre-testing requirements as well as technical processes for testing each of the test cases. Having detailed test plans reduced our project risk and provided a framework for ensuring a consistent level of data quality across hospital activations.

Conducting System Testing of the Solution: We performed multiple levels of testing to ensure the quality of our end-to-end data collection processes. We began by unit testing each process component to ensure that it performed according to its design specifications. After successfully completing unit testing we strung together all of our process components (see Figure 3 above) into a system test. The system test was guided by our test plans and scenarios.

System testing was performed in multiple test cycles. For each test cycle information was entered into a hospital's test Meditech HIS that covered the test cases within that test cycle. These data were then flowed through our Biosurveillance Integrator to ensure that that it could properly process and transform those data into the biosurveillance messages. Upon completion of the system test cycle testing results were documented in a testing report spreadsheet. This test report also contains information about the test cycle, the testing environment, the facilities being tested, and any other appropriate configuration data required during the testing process. Following the successful system testing for a hospital, the data activation activities were scheduled and a hand-off document containing system support responsibilities and contact information was created.

The result of these activities is data activation to make the new syndromic surveillance data feeds available to end users and to prepare the production systems support team for assuming responsibility for the newly implemented data feeds.

\section{Results}

NW-PHIE used a structured activation process that ensured syndromic surveillance data activations were coordinated along the entire processing chain from hospital, to INHS HIE personnel, to local, state and federal public health - resulting in standardized clinical data being made useful to public health and the needs of state DOH and LHJs being met.

Activities related to the activation of the syndromic surveillance data feeds consist of the following three steps: 
1. Preparation for Activation - including completing certification of data feed based on testing/acceptance protocols; completing user and operational support training; obtaining signoff from the facility to activate the data feed; scheduling the activation and notifying data recipients of activation schedule; distribution of activation checklist, resource assignments, and technical documentation for system support; completing preparation of the production environment to receive the data; and conducting a pre-activation meeting to verify activation task assignments/status.

2. System Activation - including completion of necessary system backups/recovery plans; conducting a checkpoint meeting prior to start of deployment; executing the development plan for migrating software to production environment; performing verification of an initial batch of data related to activation; validating a data sample from existing data feeds to confirm changes have not impacted existing messages; conducting a checkpoint meeting prior to activation of data feed in production; enabling the data feed and monitoring successful transmission and receipt to data consumer systems; and documenting issues and notifying stakeholders and users of activation.

3. Support/Maintenance - including monitoring the data feed for initial period, based on volumes/frequency of data; performing data quality analysis on large data sampling for possible vocabulary exceptions and issues; reviewing the activation plan and documenting lessons learned for incorporation into future activations; transferring responsibilities of monitoring and support to performing organization; and providing hands-on assistance for level two support by development team.

WA DOH receives the Biosurveillance HL7 messages in 15 minute increments. These messages are stored into a data queue that is immediately indexed into an HL7-centric database schema. Information for a single patient is split into many discrete biosurveillance HL7 messages triggered by events at the facility such as registration, a lab order, a lab result, etc. In order to make clinical data useful for population health purposes several steps need to be followed. First, the individual messages need to be reassembled into a longitudinal, comprehensive view of a visit encounter. This is done by using the system generated visit identifier to link ADT, lab orders, results, vital signs, etc. to reconstruct the comprehensive data for a visit. Next, the encounters need to be classified according to surveillance criteria, and then counted over a fixed time interval, and paired with appropriate denominator, such as total visit volume or catchment population. Each component can be seen in the Entity Relationship diagram in Figure 4 which details how the longitudinal visit record is generated from line-level HL7 messages. 


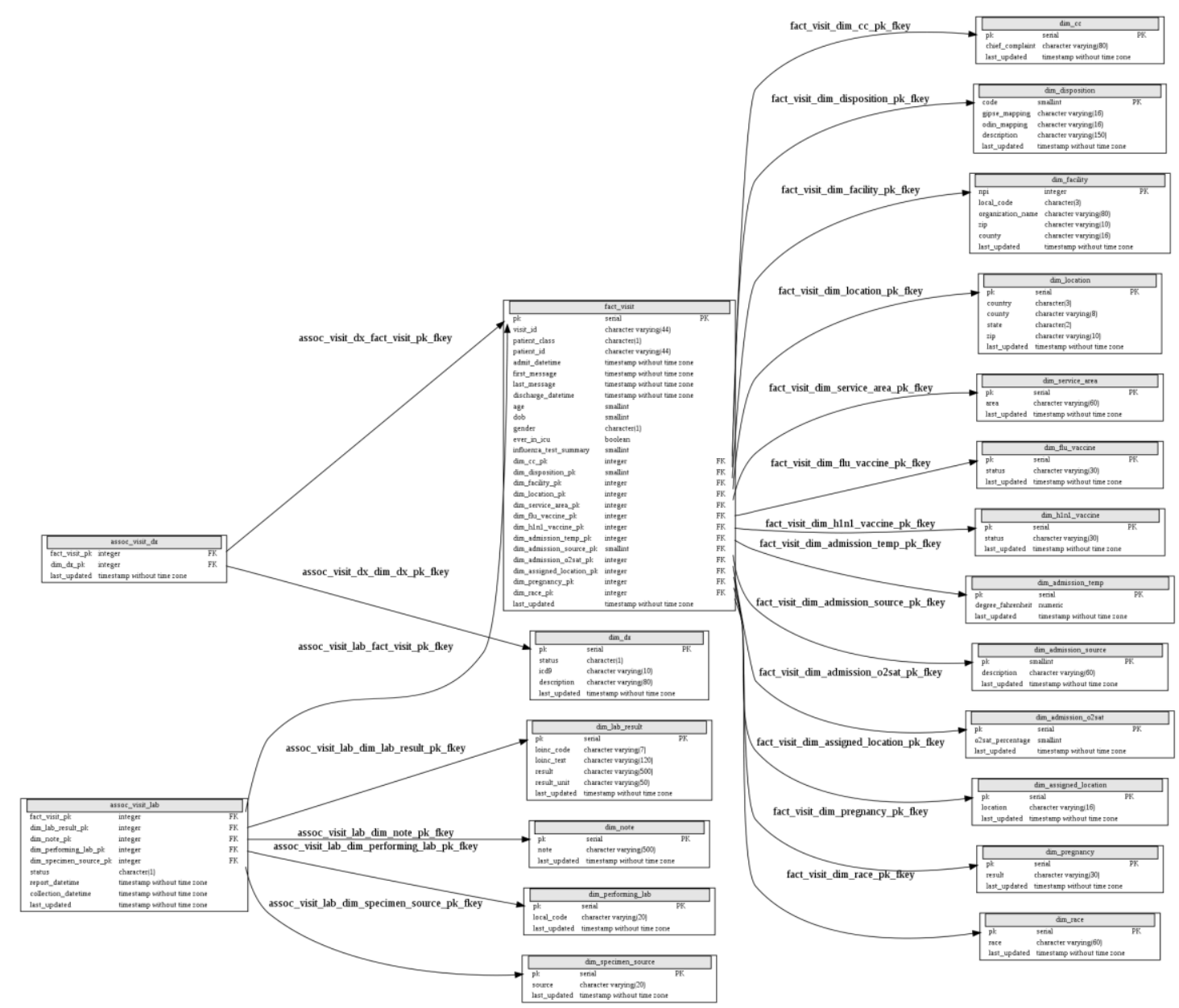

Figure 4. Entity Relationship Diagram

Using indicator definitions such as those listed in Table 2, encounters are classified according to surveillance criteria.

Table 2. Indicator Classifications and Definitions (partial example)

\begin{tabular}{|c|c|c|c|}
\hline $\begin{array}{l}\text { Indicator } \\
\text { Identifier }\end{array}$ & Indicator Name & $\begin{array}{l}\text { MBDS } \\
\text { Field(s) }\end{array}$ & Indicator Definition \\
\hline 1001 & $\begin{array}{l}\text { Influenza-like } \\
\text { Illness chief } \\
\text { complaint (ILI-1) }\end{array}$ & $\begin{array}{l}\text { Chief } \\
\text { Complaint }\end{array}$ & $\begin{array}{l}1003 \text { OR (1004 AND }(1005 \text { OR } \\
1006))\end{array}$ \\
\hline 1002 & $\begin{array}{l}\text { Influenza-like } \\
\text { Illness chief } \\
\text { complaint (ILI-2) }\end{array}$ & $\begin{array}{l}\text { Chief } \\
\text { Complaint }\end{array}$ & 1004 AND (1005 OR 1006) \\
\hline 1003 & $\begin{array}{l}\text { Influenza chief } \\
\text { complaint } \\
\text { (ICC-1) }\end{array}$ & $\begin{array}{l}\text { Chief } \\
\text { Complaint }\end{array}$ & $\begin{array}{l}\text { Flu-like } \\
\text { influenza } \\
\text { influenza } \\
\text { intestinal } \\
\text { spinal } \\
\text { stomach flu) }\end{array}$ \\
\hline
\end{tabular}




\begin{tabular}{|c|c|c|c|}
\hline $\begin{array}{l}\text { Indicator } \\
\text { Identifier }\end{array}$ & Indicator Name & $\begin{array}{l}\text { MBDS } \\
\text { Field(s) }\end{array}$ & Indicator Definition \\
\hline 1004 & $\begin{array}{l}\text { Fever chief } \\
\text { complaint } \\
\text { (FCC-1) }\end{array}$ & $\begin{array}{l}\text { Chief } \\
\text { Complaint }\end{array}$ & $\begin{array}{l}\text { Chills } \\
\text { fever } \\
\text { febrile } \\
\text { rigors } \\
\text { temperature }\end{array}$ \\
\hline 1005 & $\begin{array}{l}\text { Cough chief } \\
\text { complaint } \\
(\mathrm{CCC}-1)\end{array}$ & $\begin{array}{l}\text { Chief } \\
\text { Complaint }\end{array}$ & Cough \\
\hline 1006 & $\begin{array}{l}\text { URI chief } \\
\text { complaint } \\
\text { (UCC-1) }\end{array}$ & $\begin{array}{l}\text { Chief } \\
\text { Complaint }\end{array}$ & $\begin{array}{l}\text { Achy throat OR epiglottitis OR } \\
\text { head pressure OR inflamed throat OR } \\
\text { nasal congestion OR pharyngitis OR } \\
\text { rhinitis OR runny nose OR } \\
\text { scratchy throat OR sinus pain OR } \\
\text { sinusitis OR sneeze } \\
\text { sore throat OR stuffy nose OR } \\
\text { tonsillitis OR upper respiratory } \\
\text { infection } \\
\text { burning in throat OR inflamed tonsil } \\
\text { OR } \\
\text { throat pain OR pharyngotonsillitis OR } \\
\text { strep OR swollen throat OR } \\
\text { swollen tonsil OR swollen uvula OR } \\
\text { throat drainage OR throat dry OR } \\
\text { throat infection OR throat irritation OR } \\
\text { throat itch OR throat tingling OR } \\
\text { tonsil infection OR tonsil pain OR } \\
\text { cold }\end{array}$ \\
\hline
\end{tabular}

Encounters are then aggregated over a fixed time interval and paired with appropriate denominator, such as total visit volume or catchment population. From these data absolute counts and rates of illness within the patient population are obtained.

Within Washington State, LHJs initiate outbreak investigation and WA DOH assists LHJ staff by facilitating testing done through the state public health laboratory and CDC as well as provides coordination and staffing to support outbreaks that involve multiple LHJs. WA DOH is also tasked with understanding the state-wide implications of outbreaks and providing state-wide reporting.

Critical to timely management of outbreaks is early identification of the outbreak along with case identification. Many outbreaks of public health interest are not mandated to be reported to public health based on state notifiable disease reporting laws. Common pathogens that are not reportable as isolated cases include influenza, varicella, RSV, and norovirus. NW-PHIE's automated syndromic surveillance systems sends de-identified data to the WA DOH and create summary statistics to identify outbreaks. The de- 
identified syndromic surveillance data provided to the WA DOH is sufficient to allow the state to perform its state-wide monitoring and reporting responsibilities.

Once an outbreak has been identified LHJs oftentimes need to re-identify patients to perform their outbreak investigation. This re-identification is performed by LHJ personnel calling the Health Information Manager (HIM) at the facility and providing the system generated biosurveillance linker ID. The HIM logs on to the Biosurveillance Integrator and queries to find the medical record number and name of the patient associated with the biosurveillance linker ID. This information is provided to the LHJ to assist in their outbreak investigation. This rapid identification of outbreaks and identification of patients associated with outbreaks provides LJHs with a needed head start on containing the outbreak by performing case and contact investigations.

\section{Discussion and Conclusion}

The experience of the NW-PHIE project provides an organizational and operational roadmap for implementing a successful regional HIE that ensures secure exchange and use of electronic health information between local and state public health and health care entities. Given the role HIEs are expected to play as building blocks for a proposed National Health Information Network as well as in the Office of the National Coordinator for Technology (ONC) plan for a National Health Information Exchange Model, it is important to capture the lessons learned from the NW-PHIE experience. From our experience we have extracted five significant lessons we believe need to be included to achieve HIE success:

\section{Lesson 1. Contracts}

Having a contract for the NW-PHIE work that was written through a participatory and consensus process involving all key stakeholders focused our efforts by providing clear goals and deliverables. Having each stakeholder contribute to the contract enabled an investment in the NW-PHIE's success. However, it is important to note that this contract also allowed room for flexibility (see "Creativity" below). In addition, Data Use Agreement contracts provided evidence of the NW-PHIE's openness and transparency as well as respect for and protection of the core component of the NW-PHIE: data.

\section{Lesson 2. Collaboration}

A key lesson learned was the benefits of collaborating, in particular involving local and state public health as a fully participating partner at the beginning of the project. For example, after collecting the MBDS data elements, state and local epidemiologists were given access so they could provide input on system requirements, participate in tool design and have their work process needs reflected by these tools. Perhaps most importantly, once the epidemiologists had the data not only was it easier for them to see the project's value but they took the lead in fleshing out requirements. This collaboration cemented a buy-in for epidemiologists that would not have occurred if we had started with Greenfield requirements. An additional point on collaboration is that the NW-PHIE team was structured to include a blended set of technical, public health, clinical, project management, and research skills as represented by an HIE, multiple health departments, a 
systems integrator and an academic research university. The skills and assets of each group were leveraged throughout the NW-PHIE project.

\section{Lesson 3. Consensus}

Achieving consensus on clearly defined short- and long-term goals that addressed the needs and priorities of all stakeholders was a key to ultimate success. It was also important to incorporate consensus-based data sharing policies and practices. An example of this is the MBDS definition which was developed through expert opinion, informed by syndromic surveillance systems across the country and national groups sponsored by AHIC, and thus provided the NW-PHIE team with a trusted and clear definition of the data elements to collect. We also found that it was important to determine "how" and "what" was needed out of an existing EHR, as opposed to expecting the EHR to change or create new elements as needed. Achieving consensus on these requirements involved all partners.

\section{Lesson 4. Communication}

The team participated in regular bi-weekly conference calls that provided a level of governance, oversight and a forum for regular participation by all team members. These regular communications focused on the project milestones and deliverables but also allowed time for creative problem-solving. In addition, the larger HIE Grantees held regular conference calls that the NW-PHIE team was invited to participate in, which assured transparency and helped build a larger HIE community.

\section{Lesson 5. Creativity}

Being able to try out different approaches to exchanging and analyzing the data was critical. Public health benefitted from the rapid and throw-away testing "sandbox" provided by the university in which data could be analyzed and modified until solutions were tested and developed. And although we had a contract with clear goals and deliverables, there was enough flexibility in the contract for creativity and response to crisis situations (e.g. the H1N1 outbreak) and opportunities to participate in conferences and demonstrations. It is also important to note that the H1N1 public health events which occurred during the project enabled our activities to receive a higher priority within healthcare organizations and energized the project. As of publication of this article, the flexibility that rapid role out provided - in combination with the ability to academically review data and implement new features - continues.

\section{Acknowledgments}

This work was funded by the Centers for Disease Control and Prevention through the "Accelerating Public Health Situational Awareness through Health Information Exchanges" contract \#200-2008-24369. We also wish to acknowledge helpful feedback from Michael Davisson and Bryant Karras at WA State DOH; Paul Bugni and Bill Lober at UW; and Mark Springer at SRHD; along with the work of the entire NWPHIE Collaborative. 


\section{Correspondence}

Michael Trebatoski, Science Applications International Corporation, Healthcare Systems, McLean, VA. trebatoskim@saic.com

\section{Conflicts of Interest}

The authors do not report any conflicts of interest. 